\title{
Overview of the Nearby Supernova Factory
}

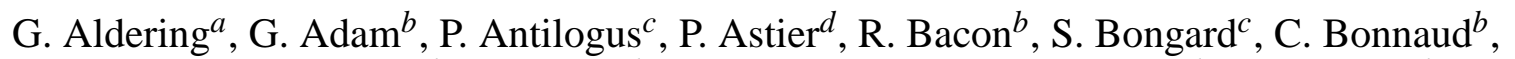 \\ Y. Copin ${ }^{c}$, D. Hardin ${ }^{d}$, F. Henault $b$, D. A. Howell ${ }^{a}$, J.-P. Lemonnier ${ }^{b}$, J.-M. Levy ${ }^{d}$,

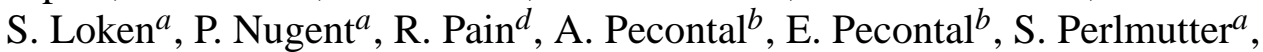 \\ R. Quimby ${ }^{a}$, K. Schahmaneche ${ }^{d}$, G. Smadja ${ }^{c}$, and W.M. Wood-Vasey ${ }^{a}$, \\ the Nearby Supernova Factory collaboration \\ ${ }^{a}$ Lawrence Berkeley National Laboratory, Berkeley CA, USA \\ ${ }^{b}$ Centre de Recherche Astronomique, Universite Lyon I and Ecole Normale Superieure, Lyon, France \\ ${ }^{c}$ Institut de Physique Nucleaire, Universite Lyon I, Lyon, France \\ ${ }^{d}$ Laboratoire de Physique Nucleaire et de Hautes Energies, Universites Paris VI and VII, Paris, France
}

\begin{abstract}
The Nearby Supernova Factory (SNfactory) is an international experiment designed to lay the foundation for the next generation of cosmology experiments (such as CFHTLS, wP, SNAP and LSST) which will measure the expansion history of the Universe using Type Ia supernovae. The SNfactory will discover and obtain frequent lightcurve spectrophotometry covering 3200-10000 $\AA$ for roughly 300 Type Ia supernovae at the low-redshift end of the smooth Hubble flow. The quantity, quality, breadth of galactic environments, and homogeneous nature of the SNfactory dataset will make it the premier source of calibration for the Type Ia supernova width-brightness relation and the intrinsic supernova colors used for $K$-correction and correction for extinction by host-galaxy dust. This dataset will also allow an extensive investigation of additional parameters which possibly influence the quality of Type Ia supernovae as cosmological probes. The SNfactory search capabilities and follow-up instrumentation include wide-field CCD imagers on two 1.2-m telescopes (via collaboration with the Near Earth Asteroid Tracking team at JPL and the QUEST team at Yale), and a two-channel integral-field-unit optical spectrograph/imager being fabricated for the University of Hawaii 2.2-m telescope. In addition to ground-based follow-up, UV spectra for a subsample of these supernovae will be obtained with HST. The pipeline to obtain, transfer via wireless and standard internet, and automatically process the search images is in operation. Software and hardware development is now underway to enable the execution of follow-up spectroscopy of supernova candidates at the Hawaii 2.2-m telescope via automated remote control of the telescope and the IFU spectrograph/imager.
\end{abstract}

Keywords: supernova, survey, cosmology, integral-field-unit, spectrograph

\section{PROBING DARK ENERGY WITH SUPERNOVAE}

A coherent view of the universe is emerging in which a mysterious form of "dark energy" accounts for about $2 / 3$ of the total energy density in the Universe. Direct evidence for this radical conclusion comes from distance measurements of Type Ia supernovae (SNe Ia; see Fig. 1) which indicate the expansion of the Universe is not slowing down as would be expected in a Universe filled with only matter and radiation. ${ }^{1,2}$ Further support for this result has come from recent measurements of the CMB indicating a flat universe, ${ }^{3}$ combined with determinations of $\Omega_{M} \sim 0.3$ from structure formation.

$\mathrm{SNe}$ Ia remain the most mature cosmological distance indicator, and therefore, offer the best current means of experimentally probing the properties of the dark energy. Their cosmological use was developed in the early 1990's, paving the way for the discovery of dark energy. ${ }^{1,4-10}$ Now similar developmental efforts are needed so that the next order of magnitude improvement of the experimental constraints on the properties of dark energy can be made.

Progress must be made on two fronts, at a level which cannot be pursued with existing programs alone. First a large number of nearby SNe must be observed in an appropriate fashion since they provide the fulcrum of the lever-arm needed to make cosmological inferences from high-redshift SNe observations. Furthermore, these SNe provide the critical

Correspondence: e-mail galdering@ lbl.gov; telephone 510-495-2203 

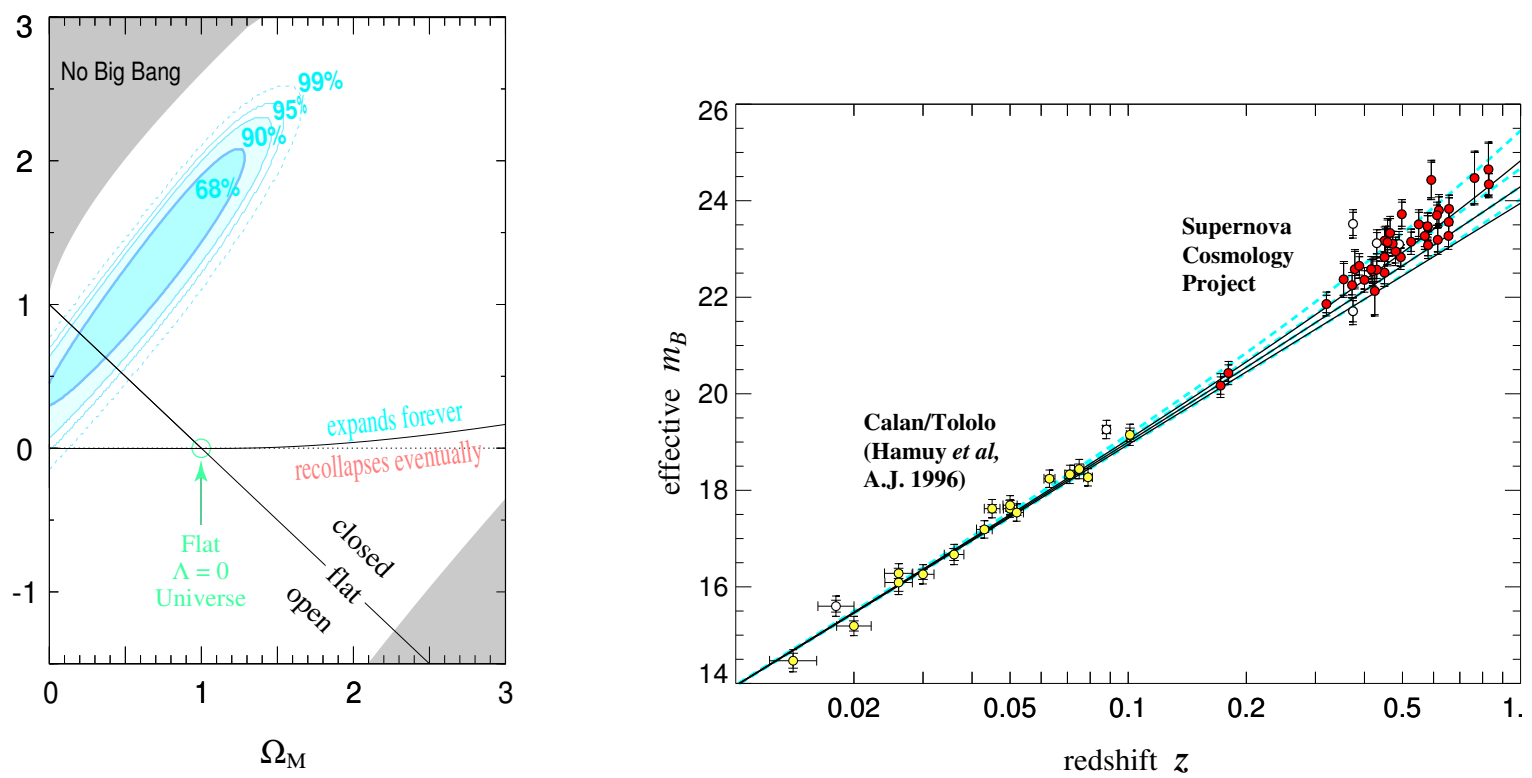

Figure 1. Cosmological constraints from Type Ia supernovae: Hubble diagram (brightness vs. redshift) and resulting constraints on $\Omega_{M}$ and $\Omega_{\Lambda}$ from 42 high-redshift Type Ia supernovae. ${ }^{1}$ At left, the $68 \%, 90 \%$, and $99 \%$ confidence regions for an unconstrained fit for $\Omega_{M}$ and $\Omega_{\Lambda}$ are shown. For a flat universe only a cosmological constant or other form of dark energy can explain the data. Even without assuming a flat universe a cosmological constant is hard to avoid for any reasonable choice of $\Omega_{M}$. At right, the Hubble diagram of the current Supernova Cosmology Project dataset of Type Ia supernovae is shown. The low-redshift SNe Ia shown are most of those currently available that are suitable for analysis using the same methods as used for the high-redshift SNe Ia.

empirical calibration of the SN lightcurve brightness-width relation, as well as providing the intrinsic SN colors needed to correct for dust extinction. At present, half the statistical uncertainty in the dark energy measurements arises from the limited pool of low-redshift SNe; we propose to increase this pool to many hundreds of well-observed nearby SNe located in the smooth Hubble flow.

Second, our understanding of the physics of the SNe Ia must be pushed to a deeper level. Presently SNe Ia appear to be excellent standardized candles, but we do not understand the details of why this is so. The chief remaining loophole in the interpretation of the SN Ia results is the possibility of a conspiratorial evolutionary effect in the explosions themselves. ${ }^{11}$ There is already some empirical data that constrains such "conspiracies," but as we move to the next generation of experiments much tighter constraints will be needed. Low-redshift supernova data provide both the necessary empirical constraints, and the matching deeper physical understanding of the SNe Ia, and are therefore a necessary complement to the ongoing high-redshift work and future projects like the SN program of the CFHT Legacy Survey (CFHTLS), the $w$ Project $(w \mathrm{P})$, the Large Synoptic Survey Telescope (LSST) and the SuperNova/Acceleration Probe (SNAP). This work to constrain evolutionary effects requires that the low-redshift SNe Ia be observed with spectroscopy over their entire lightcurves and across a wide range of galactic environments (spanning stellar age and metal content).

\section{THE NEED FOR A LARGE NEARBY SN SAMPLE}

In recognition of the importance and urgency of this work, we have begun a new experiment - the Nearby Supernova Factory (SNfactory) - designed to exploit as fully as possible the potential of low-redshift SNe. The SNfactory will concentrate on the discovery and spectrophotometric follow-up of $\sim 300-600 \mathrm{SNe}$ Ia. This is an order of magnitude more SNe than currently available samples and will yield two orders of magnitude more spectrophotometry. Moreover, our goal is produce a sample which is homogeneous, well-calibrated, and recalibratable. Such a large sample of nearby Hubble-flow SNe Ia can make significant contributions to both the statistical and systematic aspects of using SNe Ia for cosmology, as we now describe. 


\subsection{Anchoring the zero-point of the Hubble diagram}

Roughly $50 \%$ of the statistical uncertainty in the current cosmological constraints from $\mathrm{SNe}$ Ia result stems from the small number of low-redshift SNe Ia which are suitable to serve as the zero-point for the SNe Ia Hubble diagram. This zero-point is the product $L_{S N} H_{o}^{2}$, where $H_{o}$ is the Hubble constant and $L_{S N}$ is the luminosity of a standardized Type Ia supernova. For purposes of cosmology this zero-point is a "nuisance" parameter, containing no useful information while contributing to the statistical uncertainty.

The minimal criteria for low-redshift SNe Ia to be cosmologically useful is that they be in the smooth Hubble flow - so that their radial velocities reflect cosmological redshift rather than galaxy peculiar velocities; that there exists good lightcurve photometry beginning no later than 5 days after maximum light - so that determination of the peak magnitude involves little extrapolation; and that the SNe Ia are discovered in the blind-search mode used for the high-redshift SNe Ia - so that any subtle selection effects and the range of host galaxy properties are as similar as possible at both high and low redshift. The largest sample of SNe Ia satisfying these minimal criteria are from Ref 7, and are shown in Fig. 1. One can see that the cosmologically useful nearby SNe Ia are actually outnumbered by the high-redshift SNe Ia!

A number of groups are planning much larger, more comprehensive, experiments using high-redshift SNe Ia to probe the nature of dark energy. CFHTLS expects to discover and follow-up 300-600 high-redshift $(0.3<z<0.9)$ SNe Ia over five years beginning in 2003. The $w \mathrm{P}$ search at the CTIO expects to find $2000.15<z<0.75 \mathrm{SNe}$ Ia over the next five years. These experiments have the potential to measure the effective time-averaged equation of state, $w=p / \rho$, of the dark energy, with a level of precision that will begin to test whether dark energy is due to something other than Einstein's cosmological constant (for which $w=-1$, independent of time). Further down the road, the SuperNova/Acceleration Probe will begin the measurement of time variations in the equation of state - the next generation of tests for dark-energy that differs from the simple cosmological constant. These experiments will rely very heavily on the a dataset such as the SNfactory's in order to calibrate $L_{S N} H_{o}$.

This is dramatically illustrated in Fig. 2 for the case of 300 CFHTLS SNe. CFHTLS will attempt to rule out a cosmological constant by examining the case of a constant effective equation of state, $w(z)=$ constant $=w_{0}$. In the example shown, for a nominal $w_{0}=-0.8$ the high-redshift SNe Ia alone still allow $w_{0}=-1$ at the $68 \%$ confidence level (projection of solid contour). However, addition of 300 SNe Ia from the SNfactory allows a clear rejection of $w_{0}=-1$ (projection of dashed contour). This is the result of the $S N$ factory constraint on $L_{S N} H_{o}^{2}$. The $S N$ factory is also essential for getting the best statistical results for a SNAP measurement of $w(z)$. $w(z)$ is often expressed to linear order as $w(z)=w_{0}+w_{1} z$. The projection of the solid contours of Fig. 3 give the $68 \%$ confidence region for $w_{0}$ or $w_{1}$ for a $S N A P$-like dataset of $2000 \mathrm{SNe}$ Ia with $0.3<z<1.7$. In this example, SNAP does not rule out $w_{0}=-1, w_{1}=0$, that is, a cosmological constant is still allowed. However, addition of the SNfactory SNe Ia allows a clear rejection of a cosmological constant and significantly improves the measurement of $w_{0}$. In the general case, the SNfactory dataset typically halves the uncertainty in $w_{0}$ by measuring $L_{S N} H_{o}^{2}$ to $1 \%$. Thus, is it clear that the SNfactory is a necessity if the high-redshift supernova cosmology experiments are to realize their full potential.

\subsection{Calibration of the Luminosity-Lightcurve Width Relation}

The slope, $\alpha$, of the relation between SN Ia intrinsic luminosity and lightcurve width has been determined from only a relatively small $(\sim 30)$ number of Hubble-flow SNe Ia. Each of these SNe Ia has an intrinsic peak-brightness uncertainty of about $10 \%$ and measurement errors which are comparable after host-galaxy extinction correction. Moreover, the population of SNe Ia with narrow or wide lightcurves is small, thus limiting the lever-arm available to measure $\alpha$. As a result, $\alpha$ is determined to only about $25 \%{ }^{12}$ This doesn't effect individual SNe too greatly because most $\mathrm{SNe}$ are clustered around the typical lightcurve width. However, for the future large high-redshift SNe samples, where the probative value comes from averaging, there exists the potential for $\sigma_{\alpha}$ to become an important source of statistical uncertainty since it is a correlated uncertainty for all the SNe. The large SNfactory dataset should reduce the uncertainty on $\alpha$ by at least a factor of three.

\subsection{Calibration of Intrinsic Colors for Dust Extinction Correction}

Correction of SN brightnesses for host-galaxy dust extinction involves a comparison of the measured color (usually at maximum light) of a new SN with colors of SNe Ia which are extinction-free (e.g., those in elliptical galaxies, which are 


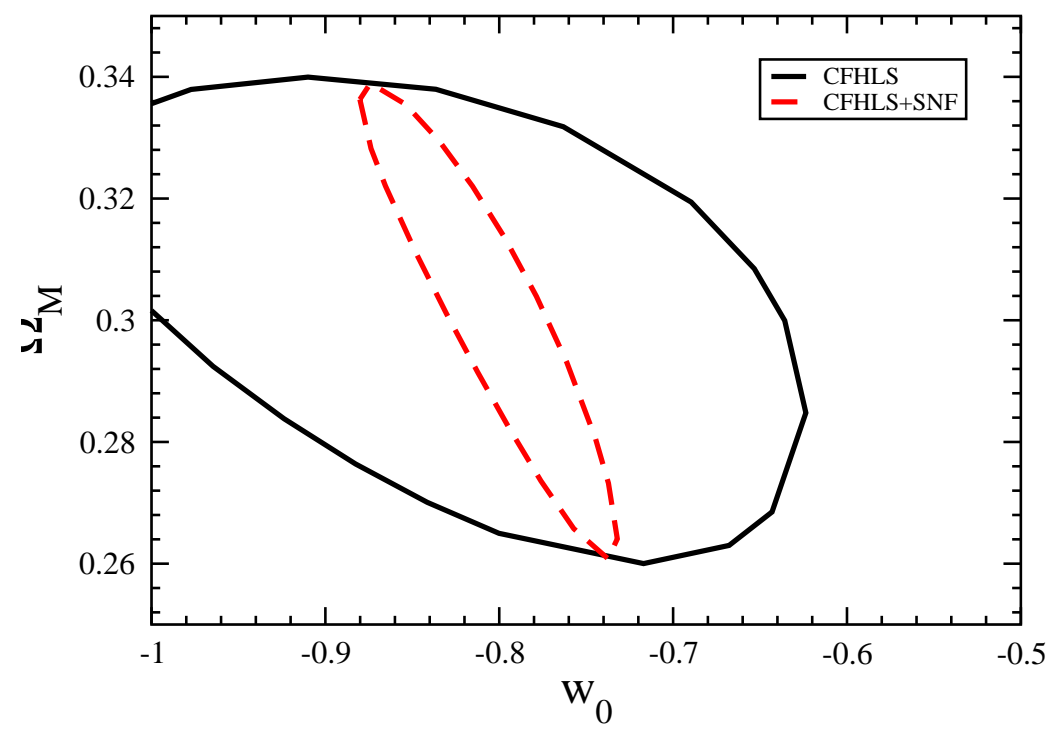

Figure 2. The importance of low-redshift SNe Ia for near-future dark energy probes: The impact of adding 300 SNfactory SNe Ia to a representative sample of 300 high-redshift $(0.3<z<0.7)$ SNe Ia, such as the CFHTLS or $w \mathrm{P}$ might obtain over the next 5 years is demonstrated. The projection of the solid contour is the $68 \%$ confidence region in the $\Omega_{M}-w_{0}$ plane from the high-redshift survey alone, while the projection of the dashed contour is the $68 \%$ confidence region when the SNfactory SNe Ia are added. In this example the addition of the SNfactory SNe Ia allows elimination of a cosmological constant model $\left(w_{0}=-1\right)$. A flat universe and a prior of \pm 0.04 on $\Omega_{M}$ has been assumed. When comparing to Fig. 3 note that here $w_{1}$ is set to zero and therefore contributes no uncertainty; if $w_{1}$ were allowed to float the uncertainty in $w_{0}$ would be much larger. Courtesy E. Linder, R. Miquel, and D. Huterer.

mostly free of dust). The change in color must be multiplied by 4.1 to obtain the extinction-corrected brightness. These intrinsic colors are a function of lightcurve epoch and depend on whether a SN is intrinsically over- or under-luminous.

The current uncertainty in the intrinsic (dust-free) colors of SNe Ia is not negligible. Only about $10 \%$ of all host galaxies are ellipticals, so the number of calibrating SNe Ia is small. Moreover, few of those SNe Ia are in the smooth Hubble-flow, where the effects between SN color and brightness due to dust and intrinsic luminosity can be separated. As a result, the uncertainty in the intrinsic SN Ia colors is one of the dominant uncertainties in the current cosmology measurements. Note that this is a correlated uncertainty in the calibration of the intrinsic colors of all SNe Ia, so it does not average out as larger samples of high-redshift SNe Ia are obtained, unlike the color measurement errors of each individual high-redshift SN Ia. The SNfactory spectrophotometric lightcurve measurements are designed to greatly improve this calibration of intrinsic colors, making it possible to take advantage of the large statistics from the next generations of high-redshift SN Ia projects.

\section{4. $K$-corrections}

Because SNe Ia are observed over a range of redshifts, in the restframe of the SN any filter used to obtain an image will not exactly match the standard $B$-band filter. As a result, the brightness of a SN Ia will be affected by spectral features which are either included or excluded due to filter mismatch and must be corrected. This " $K$-correction" requires knowledge of the SN spectrum and the photon response of the instrument. ${ }^{13,14}$ For high-redshift SNe Ia the spectrum is usually only available from around the time of maximum light, whereas each photometry point along the SN lightcurve requires its own $K$-correction. Thus, the appropriate spectrum to be used for $K$-corrections at other epochs on the lightcurve must be inferred from the spectra of low-redshift analogs. The choice of the best analog relies on comparison of the 


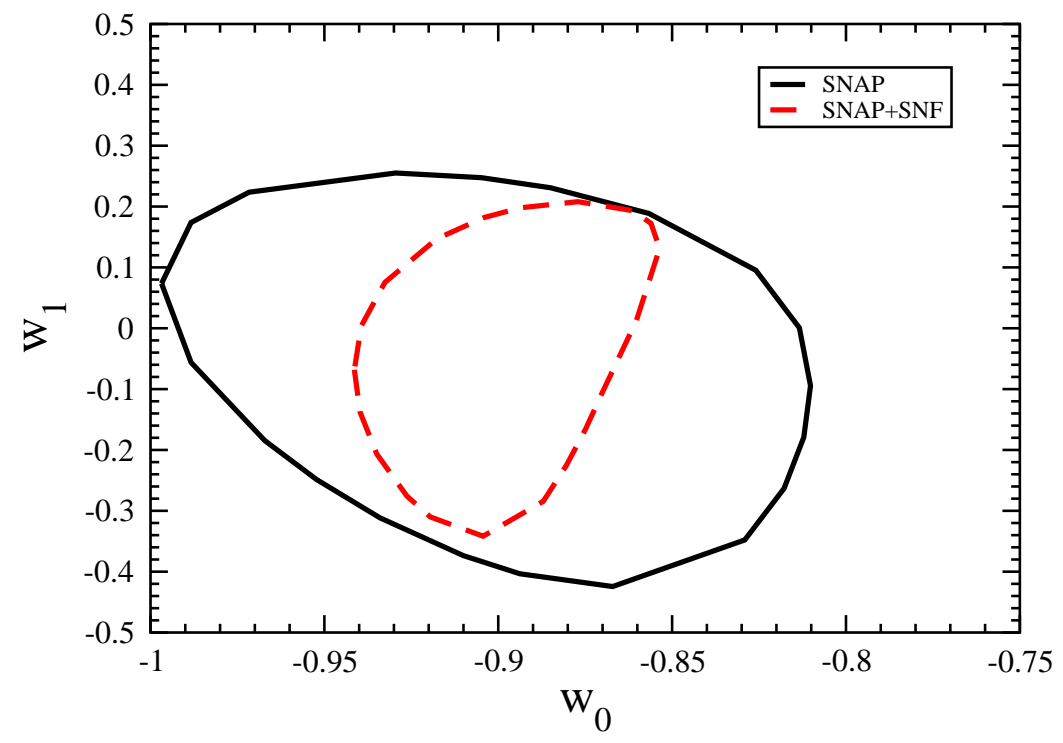

Figure 3. The importance of low-redshift SNe Ia for next-generation dark energy probes: The impact of adding 300 SNfactory $\mathrm{SNe}$ Ia to a more ambitious high-redshift SNe Ia experiment such as SNAP is demonstrated. The projection of the solid contour gives the $68 \%$ confidence region from SNAP alone, while the projection of the dashed contour is the $68 \%$ confidence region when the SNfactory SNe Ia are added. In this example the addition of the SNfactory SNe Ia allows elimination of a cosmological constant model $\left(w_{0}=-1, w_{1}=0\right)$. A flat universe has been assumed and a prior of \pm 0.04 on $\Omega_{M}$ has been imposed. Courtesy E. Linder, R. Miquel, and D. Huterer.

maximum-light spectra and the colors of the low- and high-redshift SNe (it could also depend on additional inputs, such as the lightcurve shape). The better the analog, the better the accuracy of the $K$-correction.

The SNfactory's spectral timeseries will allow synthetic photometry, thereby eliminating errors in the $K$-corrections for SNfactory SNe. This will allow excellent calibration of SNe Ia standardization relations. Moreover, the large SNfactory sample will vastly increase the number of analogs available for the $K$-correction of high-redshift SNe Ia. Thus, one can see that these gains - although merely technical - have the power to significantly improve the results from supernova cosmology experiments.

All the above steps are needed for the proper application of the current standardization methods used by all the groups who do cosmology with Type Ia supernovae. Furthermore, the reader will have noted that these various calibration and correction steps are not independent: $K$-corrections must be applied to get the $\mathrm{SN}$ color, which is then used to determine the dust extinction, after which $\alpha$ and $L_{S N} H_{o}^{2}$ can be determined. Therefore a large homogeneous dataset is required to separate-out the contributions of these various effects. In particular, a large-scale search is necessary in order to find the rarer (elliptical host galaxies, large/small lightcurve width, etc.) events which provide leverage for the calibration.

\subsection{Converting Systematic Uncertainties into Statistical Uncertainties}

Improvement of systematic uncertainties is as - or even more - important than improving the statistical uncertainties just discussed. In particular, we now want to scrutinize the SNe Ia closely enough that we can find any existing secondorder differences that are not already parameterized by the lightcurve width vs. luminosity relation. Well-observed nearby $\mathrm{SNe}$ Ia, especially in host galaxies spanning a wide range in star-formation histories, are essential for hunting for such possible second-order systematic trends and the observables that could constrain them.

By measuring key spectral ${ }^{15,16}$ and lightcurve features for each SN the physical conditions of the explosion can be tightly constrained, making it possible to recognize sets of $\mathrm{SNe}$ with matching initial conditions. The current theoretical 
models of SN Ia explosions are not sufficiently complete to predict the precise luminosity of each SN, but they are able to give the rough relationships between changes in the physical conditions of the $\mathrm{SNe}$ (such as opacity, metallicity, fused nickel mass, and nickel distribution) and changes in their peak luminosities. The SNfactory spectral timeseries will allow us to empirically calibrate these relationships between changes in the physical conditions of the $\mathrm{SNe}$ and changes in their peak luminosities. The large sample of SNfactory SNe will be important in recognizing the signature of any new SN sub-types, which could in turn signal the existence of multiple progenitor scenarios.

\section{IMPLEMENTATION OF THE SNFACTORY}

\subsection{Baseline Program}

Our previous experience with nearby supernova campaigns ${ }^{17,18}$ (which have discovered over 60 nearby SNe in all) has shown us that automation and tight coordination of the search and follow-up stages, including dedicated and optimized follow-up instrumentation, are absolutely essential to build a large sample of well-observed and well-calibrated supernovae. In addition, it has become increasingly clear that extensive spectroscopy coupled with photometry is needed to understand the SNe Ia physics and exploit this distance indicator to its fullest.

The baseline SNfactory program is to obtain spectrophotometric lightcurves covering 3200-10000 for a minimum of 300 nearby Type Ia SNe spanning as wide a range in SN parameter space as possible. These SNe Ia will be in the redshift range of $0.03<z<0.08$ - not so far as to require excessive amounts of telescope time (follow-up time goes roughly as $z^{4}$ ), yet far enough so that host galaxy peculiar velocities will contribute little to the error budget. The goal is to discover the SNe Ia as soon after explosion as possible; for the NEAT flux limit (roughly 22nd magnitude in V) and observing cadence we expect to easily find most SNe Ia 10-15 days before maximum light. SNe Ia spectra change noticeably on time-scales of 5-7 days, so we will attempt to obtain spectrophotometry every 3-5 days from -15 days through +45 days around maximum light. This follow-up cadence should yield roughly 15 spectra for each SN. At late-times some of the nearer SNe also will be observed bimonthly at late times in synthetic-photometry mode in order to better constrain positron escape models. ${ }^{19}$ Each spectrum will require 10-40 minutes to achieve adequate signal-tonoise, depending on SN brightness, atmospheric image quality, and sky brightness. Taking into account the overhead for screening of unsuitable SNe (e.g. those having the wrong type or redshift), acquisition, readout, calibration, and weather we expect to intensively study $\sim 100$ SNe Ia per year. Roughly a year after the initial observations a "final reference" spectrum will be obtained so that the host galaxy light superimposed on the previous year's SN light can be subtracted.

A key difference between the SNfactory and other major nearby SN searches is its use of ultra-wide-field CCD mosaic imagers to conduct blind - rather than targeted - searching. This search methodology is just like that employed to discover high-redshift SNe. This distinction is important since the targeted approach misses an important subset of supernovae (such as the overluminous Type Ia, SN1999aw, ${ }^{20}$ or the hypernova, SN1999as ${ }^{21,}{ }^{22}$ ), which have intrinsically faint host galaxies barely visible or invisible on the POSS. Our approach is also more efficient because each one of our images contains galaxy luminosity equivalent to about $100 L^{*}$ galaxies in the nearby smooth Hubble-flow, whereas targeted searches contain only one galaxy per image (and even those galaxies generally have $z<0.03$ ).

In addition, the SNfactory will closely coordinate discovery and follow-up observations, eliminating the delays and spotty early-lightcurve coverage which is now typical. It is expected that with the SNfactory detailed follow-up of supernova candidates can begin within as little as $3 \mathrm{hrs}$ of the discovery observations. Finally, SNfactory follow-up observations will use an integral field unit spectrograph, data from which can be used to construct both detailed flux-calibrated spectra and broadband images. This dataset will also eliminate several limitations (wavelength bandpass mismatch, wavelengthdependent slit losses, etc.) of all other currently available instrumentation used to study supernovae.

\subsection{Discovery}

Discovery of $\mathrm{SNe}$ at low redshift operates in a different regime than $\mathrm{SNe}$ searches at high redshift because at high redshift a few wide fields totaling several square degrees monitored over a year will contain many SNe, while at low redshift even the widest-field cameras will have substantially less than one SN per year. Thus, while at high redshift the search and follow-up can be combined to achieve a substantial multiplex advantage using one telescope and imager, at low redshift the optimal telescopes and instrumentation for the discovery stage are very different than for the follow-up stage.

The SNfactory is searching for supernovae using CCD images obtained by JPL's Near Earth Asteroid Team (NEAT). The properties of the imagers used by NEAT are listed in Table 1. In 15 months of operation the first Palomar imager has 
Table 1. NEAT Search Facilities

\begin{tabular}{|l|ccc|}
\hline \hline Site: & Haleakala & Palomar I & Palomar II \\
Aperture: & $1.2 \mathrm{~m}$ & $1.2 \mathrm{~m}$ & $1.2 \mathrm{~m}$ \\
Imager Format: & $4 \mathrm{k} \times 4 \mathrm{k}$ & $3 \times 4 \mathrm{k} \times 3 \mathrm{k}$ & $112 \times 2.4 \mathrm{k} \times 0.6 \mathrm{k}$ \\
Imager Scale: & $1.33^{\prime \prime} /$ pixel & $1.01^{\prime \prime} /$ pixel & $0.87^{\prime \prime} / \mathrm{pixel}$ \\
Field of View: & $1.5^{\circ} \times 1.5^{\circ}$ & $1.1^{\circ} \times 3.4^{\circ}$ & $2.3^{\circ} \times 4.0^{\circ}$ \\
Filters: & open & open & open \\
Exposures: & $3 \times 20 \mathrm{sec}$ & $3 \times 60 \mathrm{sec}$ & TBD \\
Readout: & $20 \mathrm{sec}$ & $20 \mathrm{sec}$ & $30 \mathrm{sec}$ \\
Nightly Coverage: & $300 \square^{\circ}$ & $500 \square^{\circ}$ & $\left(1000 \square^{\circ}\right)$ \\
Period: & Mar $2000-$ & Apr. 2001 - Aug. 2002 & $\sim$ Sept. 2002 - \\
Data (compressed): & 12 Gbyte/night & 40 Gbyte/night & $(80 \mathrm{Gbyte} / \mathrm{night})$ \\
\hline \hline
\end{tabular}

already surveyed over 20,000 square degrees of sky. Each patch of sky is revisited frequently (about every 6 days, since this is the "refresh rate" for NEA's); this enables early discovery — and hence early lightcurve coverage — and helps eliminate Malmquist bias. The imaging data are compressed and transferred to the National Energy Research Science Center (NERSC) at LBNL and archived on a 2 Pbyte tape vault. In the case of the Haleakala data, the high-speed internet connection between the Air Force Maui Supercomputer Center and NERSC is used. In the case of Palomar, it was necessary to set-up a custom dedicated $48 \mathrm{Mbs}$ wireless internet connection to relay the data from Palomar to the San Diego Supercomputer Center (SDSC), and then send the data on to NERSC via the Energy Sciences Network (ESnet). The images are processed and subtracted to search for SNe using the 390+ node Parallel Distributed Systems Facility (PDSF) at NERSC. Candidate transients are inspected by human scanners. In recent test runs, 7 certain SNe and several probable SNe have been discovered. ${ }^{23,24}$ An important goal of the SNfactory is to make the selection of supernova candidates quantitative and traceable; without this, supernova rates and peculiarity fractions are extremely difficult to calculate accurately.

\subsection{Follow-up — Lightcurves and Spectroscopy}

Candidate supernovae found in the NEAT images must first be screened with spectroscopy to confirm the supernovae and reveal its type (Ia, II, Ib, Ic) and redshift. Traditionally, timely supernovae spectra require target-of-opportunity observations, and extensive spectroscopy beyond the initial confirmation spectrum are rare. Follow-up usually consists of $B V R I$ optical photometry. Using a integral field unit on a two-channel (blue \& red) optical spectrograph, the SNfactory's SuperNova Integral Field Spectrograph (SNIFS ) equipped with LBNL's red-enhanced CCD's ${ }^{25}$ (see Fig. 5), will allow spectroscopy of supernovae at all epochs. The operating principle of the microlens integral field spectrographs is described in Refs 26 and 27. Because these spectra will be spectrophotometric, $U B V R I Z$ photometry can be synthesized from these spectra, without the uncertainties due to instrumental photometric color terms and $K$-corrections. SNIFS will retain one advantage of the traditional approach, which allows surrounding field stars to be used for flux scaling when conditions are non-photometric, by also having an imager which integrates on the field with a multiple-bandpass filter immediately surrounding each supernova using the exact same exposure as the integral field unit spectroscopy.

\subsection{Details of $S N I F S$}

SNIFS continuously covers a $6^{\prime \prime} \times 6^{\prime \prime}$ field of view with a sampling of $0.4^{\prime \prime}$ using a $15 \times 15$ element fused silica microlens array (MLA) being manufactured by LIMO. The scale is set by an enlarger ahead of the MLA. The MLA is followed by a masking array which rejects scattered light and light from the lenslet interstices. Tests with the MLA on OASIS at CFHT indicate that with this baffling, photometry good to $1 \%$ can be achieved (even with OASIS's epoxy MLA).

A dichroic divides the light into blue and red channels, which are operated simultaneously. The optics for each channel consists of a collimator, grism, and camera, being manufactured by SESO. These generate spectra on a thinned, low-readout noise $15 \mu \mathrm{m}$ pixel Marconi $2 \mathrm{k} \times 4 \mathrm{k}$ CCD in the blue and a $2 \mathrm{k} \times 4 \mathrm{k} 15 \mu \mathrm{m}$ pixel LBNL fully-depleted highresistivity $n$-type CCD in the red (see Fig. 5), at resolutions of $4.8 \AA$ and $6.0 \AA$, respectively. The spectra from adjacent 
Table 2. SuperNova Integral Field Spectrograph Specifications

Integral Field Unit

\begin{tabular}{|ll|}
\hline Scale & $0.4^{\prime \prime} /$ lens \\
Field of View & $6^{\prime \prime} \times 6^{\prime \prime}$ \\
\hline
\end{tabular}

\begin{tabular}{|lcc|}
\multicolumn{1}{c}{ Spectrograph } & \\
\hline Channel & Blue & Red \\
Coverage & $3200-5400 \AA$ & $5200-10000 \AA$ \\
Spectral Dispersion & $2.4 \AA /$ pixel & $3.0 \AA /$ pixel \\
Grism & $3001 / \mathrm{mm} \lambda_{B}=3800 \AA$ & $2001 / \mathrm{mm} \lambda_{B}=7250 \AA$ \\
Detector & Marconi $2 \mathrm{k} \times 4 \mathrm{k}$ & $\mathrm{LBNL} 2 \mathrm{k} \times 4 \mathrm{k}$ \\
Calibration & $\mathrm{He} / \mathrm{Hg} / \mathrm{Cd}+$ flat & $\mathrm{Ne} / \mathrm{Ar} / \mathrm{Xe}+$ flat \\
\hline
\end{tabular}

Auxillary Camera \& Guider Camera

\begin{tabular}{|lc|}
\hline Scale & $0.14^{\prime \prime} /$ pixel \\
Field of View & two $4.7^{\prime} \times 9.4^{\prime}$ regions \\
Detectors & LBNL $2 \mathrm{k} \times 4 \mathrm{k}$ \\
Filters & u,g,r,i,z,extinction monitor \\
\hline
\end{tabular}

microlenses are separated by 5-7 pixels to avoid cross-contamination. Presently the Marconi CCD is being integrated into the blue channel dewar by GL Scientific, while the LBNL CCD's are undergoing packaging and testing.

The SNIFS imager consists of a $2 \mathrm{k} \times 4 \mathrm{k}$ LBNL CCD with $15 \mu \mathrm{m}$ pixels, which views the sky surrounding the spectrograph pick-off prism. The built-in guider consists of a second identical CCD. The imager and guider are used directly, without re-imaging optics. The imager is equipped with a multiple-bandpass filter to allow measurement of the relative atmospheric extinction during the spectroscopic observations. This novel feature will allow use of non-photometric nights to produce photometric spectra, and is possible because the SNfactory will return to these same fields many times allowing the imager to be used to calibrate all the field stars self-consistently over the full spectral range of SNIFS.

The overall throughput, from a photon at the top of the atmosphere through conversion into a photoelectron at the detector, is expected to average better than $20 \%$ in the spectral channels and $50 \%$ in the imaging and guiding channels. The opto-mechanical layout of SNIFS is shown in Figs. 4.

Moving parts on SNIFS are limited to a shutter, pick-off prism (feeding the spectrograph), filter wheel and focus mechanism for the collimators. In particular, note that the grisms are fixed. The electronic components consist of detector readout (3 SDSU controllers for 4 CCD's), a shutter, filter wheel, calibration lamps, collimator focus control and statusmonitoring. The CCD's are cooled to $140 \mathrm{~K}$ using a Cryotiger closed-cycle cooler from IGC Polycold Instruments, thus eliminating the need for consumable cryogens.

SNIFS will be operated at a bent-Cassegrain port of University of Hawaii 2.2-m telescope on Mauna Kea. It will be permanently mounted, and continuously available for use $20 \%$ of the time. UH astronomers will also be able to use SNIFS for their science programs. Table 2 summarizes the technical specifications for SNIFS.

\subsection{Operations}

The operation of SNIFS is intended to be fully automated. An observer/technician is needed only to prepare the telescope for observing at the start of the night. Ancillary SNfactory software will plan the observations and command the telescope to point at specific targets. The software interface to the telescope control system to execute pointing and focus adjustments exists. The software to obtain information for the data headers and as input to the control software exists, and is being refined at University of Hawaii. SNIFS and its associated software will take focus data which will be used to adjust the telescope focus, recognize star fields near requested targets using the SNIFS imager, adjust the telescope pointing to place 


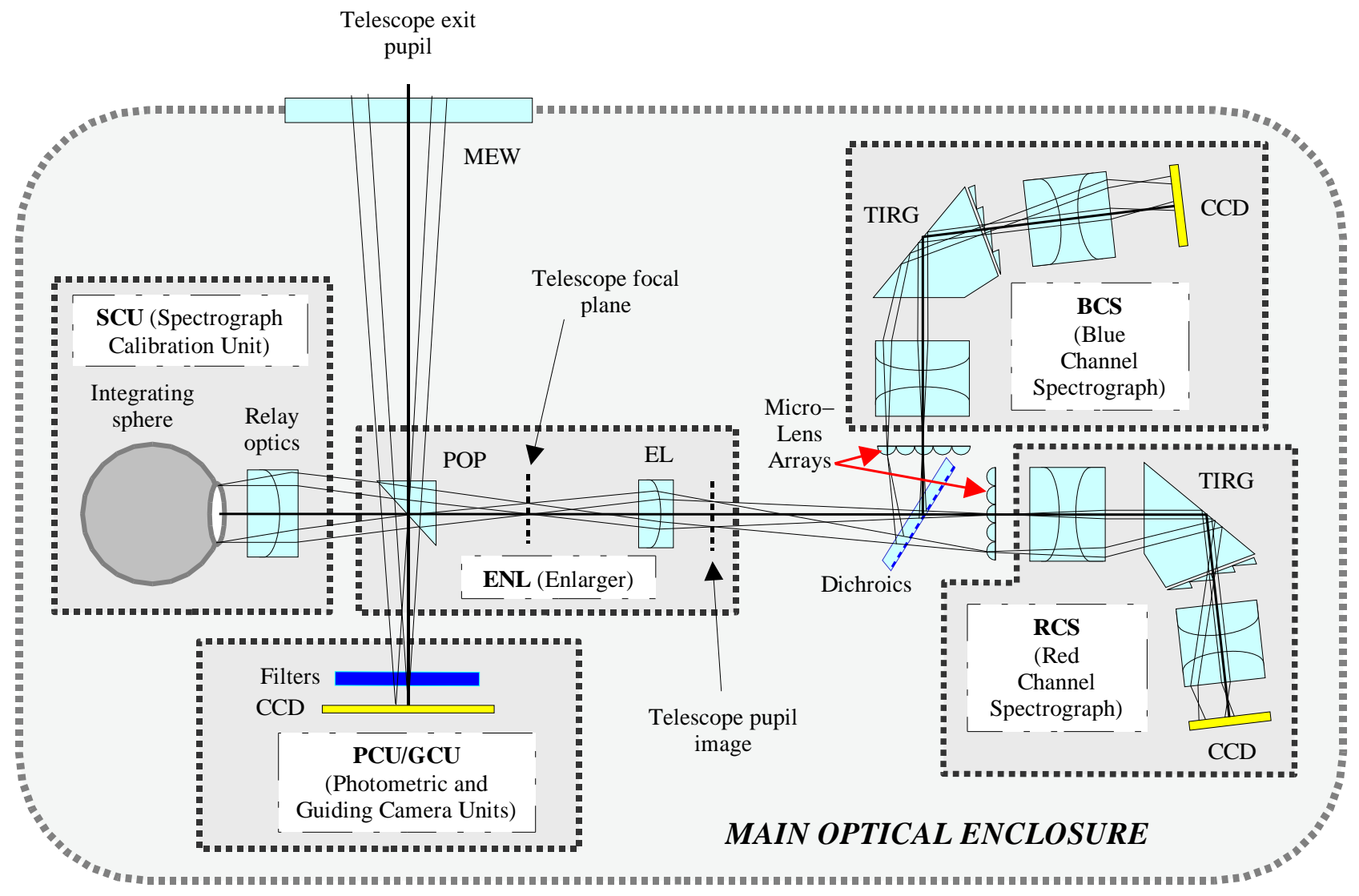

Figure 4. Break-down of SNIFS component modules: The on-axis f/10 beam from the telescope impinges on a total internal reflection pick-off prism which directs light to an enlarger. The light is then split by a dichroic, reimaged onto blue and red microlens arrays, sent through custom collimators, grism, camera, and CCD's for each of the two channels (BCS and RCS). Light from the surrounding area passes through a filter wheel and then illuminates the photometry camera (PGC); a special multiple-band filter allows the monitoring of relative atmospheric extinction during the spectroscopy observations. Off-axis light also illuminates the guider camera, providing fast guiding during spectroscopy, and allowing focusing or offsetting between exposures. On the left, a calibration unit supplies a continuum lamp and arc lamps for calibration of the spectrograph.

the desired target on the integral field unit, and acquire and guide on a suitable star. The most complicated aspect of this sequence is the automated acquisition, and we are adapting software already developed by the LBNL group for use at Keck for this purpose. After this acquisition stage, SNIFS will execute an observing sequence for the spectrograph and imager, read out the data, determine the quality of the data, and take its own calibration. As back-up, we will man command and control stations in Berkeley and France to allow manual override of the automated operations.

Once the spectra are taken, quick at-the-telescope reductions will be performed. Simultaneously, the data will be sent to the IN2P3 Computing Center (CCIN2P3) in Lyon for full processing. The processing of the IFU data into $15 \times 15 \times 2300$ element spectral data cubes will be based on an automated version of the already-existing OASIS package written by the CRAL group. This pipeline is extensive, requiring about 60 steps which can be executed in roughly 15 minutes using 3-4 $1 \mathrm{GHz}$ PIII processors. Automated spectral classification of SNe will be performed using code recently developed at LBNL.

Scheduling of the follow-up observations will be challenging, since the ultimate goal is to replace the observer at the telescope. We are presently exploring the feasibility of adapting either SPIKE or ASPEN for our use. Full environmental monitoring will be an essential component of automated operations, and is being installed and linked to the telescope 


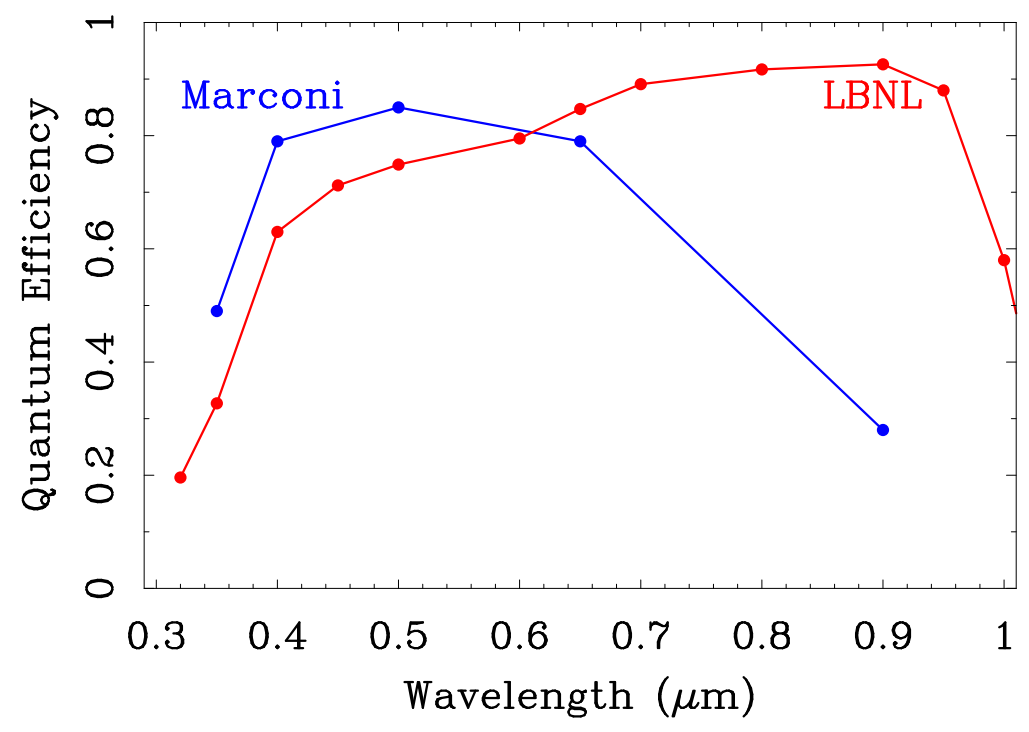

Figure 5. QE of SNIFS CCD detectors: The red solid curve shows the measured QE of LBNL CCD's which will be used in the red spectroscopic channel of SNIFS, and for the imager and guider. The blue channel of SNIFS will employ a commercial device manufactured by Marconi. The QE for our purchased Marconi device is shown with the blue curve. The spectrograph crossover between blue and red channels is at $5300 \AA$, so the net detector QE is roughly approximated by the maximum of the two curves at each wavelength.

control system at the UH 2.2-m.

\subsection{Complementary Observations}

Provided our current low-redshift SN observing program with HST is extended through future proposal cycles, the SNfactory will obtain UV spectra of a subsample of the SNe Ia using STIS. This is invaluable for glimpsing the properties of SNe Ia at wavelengths which are redshifted into the visible at high redshift. The restframe UV contains important information on the metal content of the SN atmosphere. ${ }^{28}$ Further coordinated simultaneous multi-band near-infrared imaging would allow the determination of the bolometric luminosity needed to determine the total energy budget of the $\mathrm{SNe}$, as well as providing a better determination of the amount of dust extinction suffered by each SN. ${ }^{29}$ Rest-frame near-infrared SN data are extremely scarce; our large sample of low-redshift SNe may reveal better SNe Ia standardization techniques which could be applied to high-redshift SNe Ia in the future using SNAP or the Next Generation Space Telescope (NGST). ${ }^{30}$ The SNfactory could also work to develop Type II supernovae (SNe II) as more reliable distance indicators. Since the physics and measurement methods applicable to Type Ia and Type II supernovae are so different, they will serve as valuable cross-checks on each other.

\section{CONCLUSION}

The SNfactory is intended to revolutionize all phases of experimental work on supernovae. The rate of discovery of nearby Hubble-flow will exceed the current rate by a large factor, the discovery biases will be lessened (and traceable), and the quality and quantity of follow-up data should significantly exceed that currently available. With such data, the statistical uncertainty on $w_{0}$ from the high-redshift work can be reduced by a factor of two through measurement of $L_{S N} H_{o}^{2}$, while the improved calibration of the intrinsic SNe Ia colors and $K$-corrections will reduce both the statistical and systematic uncertainty on the measurement of the standardized fluxes of the high-redshift SNe Ia. In addition, with a large sample of SNe Ia spectral timeseries from SNIFS it is expected that great strides can be made in our understanding of the underlying physics of supernovae and thereby improve them as cosmological distance indicators. Finally, the SNfactory study of the peculiar velocities of the nearest supernova host galaxies should provide competitive dynamical constraints on the value of $\Omega_{M} \cdot{ }^{31}$ 


\section{ACKNOWLEDGMENTS}

The SNfactory acknowledges support in France from the Centre National de La recherche Scientifique (CNRS) through the Institut National de Physique Nucleaire et de Physique des particules (IN2P3), the Institut National des Sciences de l'Univers (INSU) and the Programme National de Cosmology (PNC). Support in the United States is provided by the Department of Energy (DOE) Laboratory Director's Research and Development (LDRD) grant. Additional support was provided by the France-Berkeley funds. We are grateful to Hans-Werner Braun, Bud Hale and the rest of the High

Performance Wireless Research and Education Network (HPWREN) project for setting up the wireless connection to Palomar, as well as Bob Thicksten, John Henning, and others at Palomar Observatory for their help with the installation. This project could not be done without the support of the NEAT team at JPL. We would also like to thank our UC Berkeley undergraduate scanners for helping to refine the search software.

\section{REFERENCES}

1. S. Perlmutter, G. Aldering, G. Goldhaber, R. A. Knop, P. Nugent, P. G. Castro, S. Deustua, S. Fabbro, A. Goobar, D. E. Groom, I. M. Hook, A. G. Kim, M. Y. Kim, J. C. Lee, N. J. Nunes, R. Pain, C. R. Pennypacker, R. Quimby, C. Lidman, R. S. Ellis, M. Irwin, R. G. McMahon, P. Ruiz-Lapuente, N. Walton, B. Schaefer, B. J. Boyle, A. V. . Filippenko, T. Matheson, A. S. Fruchter, N. Panagia, H. J. M. Newberg, and W. J. Couch, "Measurements of Omega and Lambda from 42 High-Redshift Supernovae," Astrophys J. 517, pp. 565-586, 1999.

2. A. G. Riess, A. V. Filippenko, P. Challis, A. Clocchiatti, A. Diercks, P. M. Garnavich, R. L. Gilliland, C. J. Hogan, S. Jha, R. P. Kirshner, B. Leibundgut, M. M. Phillips, D. Reiss, B. P. Schmidt, R. A. Schommer, R. C. Smith, J. Spyromilio, C. Stubbs, N. B. Suntzeff, and J. Tonry, "Observational Evidence from Supernovae for an Accelerating Universe and a Cosmological Constant," Astron. J. 116, pp. 1009-1038, 1998.

3. C. B. Netterfield, P. A. R. Ade, J. J. Bock, J. R. Bond, J. Borrill, A. Boscaleri, K. Coble, C. R. Contaldi, B. P. Crill, P. de Bernardis, P. Farese, K. Ganga, M. Giacometti, E. Hivon, V. V. Hristov, A. Iacoangeli, A. H. Jaffe, W. C. Jones, A. E. Lange, L. Martinis, S. Masi, P. Mason, P. D. Mauskopf, A. Melchiorri, T. Montroy, E. Pascale, F. Piacentini, D. Pogosyan, F. Pongetti, S. Prunet, G. Romeo, J. E. Ruhl, and F. Scaramuzzi, "A Measurement by BOOMERANG of Multiple Peaks in the Angular Power Spectrum of the Cosmic Microwave Background," Astrophys. J. 571, pp. 604-614, 2002.

4. W. J. Couch, S. Perlmutter, H. J. M. Newburg, C. Pennypacker, G. Goldhaber, R. Muller, and B. J. Boyle, "The Berkeley / AAO Distant Supernova Search," Proc. of the Astron. Soc. of Australia 9, p. 261, 1991.

5. A. G. Riess, W. H. Press, and R. P. Kirshner, "Using Type IA supernova light curve shapes to measure the Hubble constant," Astrophys. J. 438, pp. L17-LL20, 1995.

6. S. Perlmutter, C. R. Pennypacker, G. Goldhaber, A. Goobar, R. A. Muller, H. J. M. Newberg, J. Desai, A. G. Kim, M. Y. Kim, I. A. Small, B. J. Boyle, C. S. Crawford, R. G. McMahon, P. S. Bunclark, D. Carter, M. J. Irwin, R. J. Terlevich, R. S. Ellis, K. Glazebrook, W. J. Couch, J. R. Mould, T. A. Small, and R. G. Abraham, "A Supernova at $z=0.458$ and Implications for Measuring the Cosmological Deceleration," Astrophys. J. 440, pp. L41-LL44, 1995.

7. M. Hamuy, M. M. Phillips, N. B. Suntzeff, R. A. Schommer, J. Maza, and R. Aviles, "The Absolute Luminosities of the Calan/Tololo Type Ia Supernovae," Astron. J. 112, p. 2391, 1996.

8. S. Perlmutter, S. Gabi, G. Goldhaber, A. Goobar, D. E. Groom, I. M. Hook, A. G. Kim, M. Y. Kim, J. C. Lee, R. Pain, C. R. Pennypacker, I. A. Small, R. S. Ellis, R. G. McMahon, B. J. Boyle, P. S. Bunclark, D. Carter, M. J. Irwin, K. Glazebrook, H. J. M. Newberg, A. V. Filippenko, T. Matheson, M. Dopita, W. J. Couch, and The Supernova Cosmology Project, "Measurements of the Cosmological Parameters Omega and Lambda from the First Seven Supernovae at $z>0.35$," Astrophys. J. 483, p. 565, 1997.

9. B. P. Schmidt, N. B. Suntzeff, M. M. Phillips, R. A. Schommer, A. Clocchiatti, R. P. Kirshner, P. Garnavich, P. Challis, B. Leibundgut, J. Spyromilio, A. G. Riess, A. V. Filippenko, M. Hamuy, R. C. Smith, C. Hogan, C. Stubbs, A. Diercks, D. Reiss, R. Gilliland, J. Tonry, J. . Maza, A. Dressler, J. Walsh, and R. Ciardullo, "The High-Z Supernova Search: Measuring Cosmic Deceleration and Global Curvature of the Universe Using Type Ia Supernovae," Astrophys. J. 507, pp. 46-63, 1998.

10. S. Perlmutter, G. Aldering, M. della Valle, S. Deustua, R. S. Ellis, S. Fabbro, A. Fruchter, G. Goldhaber, D. E. Groom, I. M. Hook, A. G. Kim, M. Y. Kim, R. A. Knop, C. Lidman, R. G. McMahon, P. Nugent, R. Pain, N. Panagia, C. R. Pennypacker, P. Ruiz-Lapuente, B. Schaefer, and N. Walton, "Discovery of a Supernova Explosion at Half the Age of the Universe," Nature 391, p. 51, 1998. 
11. P. S. Drell, T. J. Loredo, and I. Wasserman, "Type Ia Supernovae, Evolution, and the Cosmological Constant," Astrophys. J. 530, pp. 593-617, 2000.

12. M. M. Phillips, P. Lira, N. B. Suntzeff, R. A. Schommer, M. Hamuy, and J. . Maza, "The Reddening-Free Decline Rate Versus Luminosity Relationship for Type Ia Supernovae,” Astron. J. 118, pp. 1766-1776, 1999.

13. A. Kim, A. Goobar, and S. Perlmutter, "A Generalized $K$-Correction for Type Ia Supernovae: Comparing R-band Photometry beyond z=0.2 with B, V, and R-band Nearby Photometry," Pub. Astron. Soc. Pacific 108, p. $190,1996$.

14. P. Nugent, A. Kim, and S. Perlmutter, "K-corrections and Extinction Corrections for Type Ia Supernovae," Pub Astron. Soc. Pacific 114, pp. 803-819, 2002.

15. P. Nugent, M. Phillips, E. Baron, D. Branch, and P. Hauschildt, "Evidence for a Spectroscopic Sequence among Type 1a Supernovae," Astrophys. J. 455, p. L147, 1995.

16. P. Hoeflich, J. C. Wheeler, and F. K. Thielemann, "Type Ia Supernovae: Influence of the Initial Composition on the $\mathrm{Nu}$ cleosynthesis, Light Curves, and Spectra and Consequences for the Determination of $\Omega_{M}$ and Lambda," Astrophys. J. 495, p. 617, 1998.

17. G. Aldering, "Type Ia Supernovae \& Cosmic Acceleration,” in AIP Conference Proceeding: Cosmic Explosions, 2000 .

18. S. Perlmutter, R. A. Muller, H. J. M. Newberg, C. R. Pennypacker, T. P. Sasseen, and C. K. Smith, “A doubly robotic telescope - The Berkeley Automated Supernova Search," in ASP Conf. Ser. 34: Robotic Telescopes in the 1990s, pp. 67-71, 1992.

19. P. A. Milne, L.-S. The, and M. D. Leising, "Late Light Curves of Type Ia Supernovae," Astrophys. J. 559, pp. 10191031, 2001.

20. L.-G. Strolger, R. C. Smith, N. B. Suntzeff, M. M. Phillips, G. Aldering, P. Nugent, R. Knop, S. Perlmutter, R. A. Schommer, L. C. Ho, M. Hamuy, K. Krisciunas, L. M. Germany, R. Covarrubias, P. Candia, A. Athey, G. Blanc, A. Bonacic, T. Bowers, A. Conley, T. Dahlen, W. Freedman, G. Galaz, E. Gates, G. Goldhaber, A. Goobar, D. Groom, I. M. Hook, R. Marzke, M. Mateo, P. McCarthy, J. Mendez, C. Muena, S. E. Persson, R. Quimby, M. Roth, P. RuizLapuente, J. Seguel, A. Szentgyorgyi, K. von Braun, W. M. Wood-Vasey, and T. York, "The Type Ia Supernova 1999aw: a Probable 1999aa-like Event In a Low-Luminosity Host Galaxy," in accepted for publication in Astron. J., p. 7409, 2002.

21. R. Knop, G. Aldering, S. Deustua, G. Goldhaber, M. Kim, P. Nugent, E. Helin, S. Pravdo, D. Rabinowitz, and K. Lawrence, "Supernovae 1999as and 1999at in Anonymous Galaxies," IAU Circular 7128, p. 1, 1999.

22. K. Hatano, K. Maeda, J. S. Deng, K. Nomoto, D. Branch, P. Nugent, and G. Aldering, "Spectroscopic Analysis of Type Ic Hypernova 1999as," in ASP Conf. Ser. 251: New Century of X-ray Astronomy, p. 244, 2001.

23. W. M. Wood-Vasey, G. Aldering, and P. Nugent, "Supernova 2002dg," IAU Circular 7915, p. 2, 2002.

24. W. M. Wood-Vasey, G. Aldering, and P. Nugent, "Supernova 2002dh," IAU Circular 7916, p. 1, 2002.

25. S. E. Holland, M. Wei, K. Ji, W. E. Brown, D. K. Gilmore, R. J. Stover, D. E. Groom, M. E. Levi, N. Palaio, and S. Perlmutter, "Large Format CCD Image Sensors Fabricated on High Resistivity Silicon," in IEEE Workshop on Charge-Coupled Devices and Advanced Image Sensors, p. 239, 1999.

26. R. Bacon, G. Adam, A. Baranne, G. Courtes, D. Dubet, J. P. Dubois, E. Emsellem, P. Ferruit, Y. Georgelin, G. Monnet, E. Pecontal, A. Rousset, and F. Say, "3D spectrography at high spatial resolution. I. Concept and realization of the integral field spectrograph TIGER.," Astron. \& Astrophys. Suppl. 113, p. 347, 1995.

27. R. Bacon, Y. Copin, G. Monnet, B. W. Miller, J. R. Allington-Smith, M. Bureau, C. Marcella Carollo, R. L. Davies, E. Emsellem, H. Kuntschner, R. F. Peletier, E. K. Verolme, and P. Tim de Zeeuw, "The SAURON project - I. The panoramic integral-field spectrograph," Mon. Not. Royal. Astron. Soc. 326, pp. 23-35, 2001.

28. E. J. Lentz, E. Baron, D. Branch, P. H. Hauschildt, and P. E. Nugent, "Metallicity Effects in Non-LTE Model Atmospheres of Type Ia Supernovae,” Astrophys. J. 530, pp. 966-976, 2000.

29. K. Krisciunas, N. C. Hastings, K. Loomis, R. McMillan, A. Rest, A. G. Riess, and C. Stubbs, "Uniformity of (VNear-Infrared) Color Evolution of Type Ia Supernovae and Implications for Host Galaxy Extinction Determination,' Astrophys. J. 539, pp. 658-674, 2000.

30. W. P. S. Meikle, "The absolute infrared magnitudes of Type Ia supernovae," Mon. Not. Royal Astron. Soc. 314, pp. 782-792, 2000.

31. S. Courteau and A. Dekel, "Cosmic Flows: A Status Report," in ASP Conf. Ser. 245: Astrophysical Ages and Times Scales, p. 584, 2001. 